\title{
A Literature REVIEW ON SELF BALANCING IN LINE TWO-WHEELER (BI-CYCLE)
}

\author{
Kanav Bhatia \\ Department of Mechanical Engineering, \\ MRIIRS, Faridabad, Haryana, India
}

\author{
Ankit Singla \\ Department of Mechanical Engineering \\ MRIIRS, Faridabad, Haryana, India
}

\author{
Amit Bhatia \\ Department of Industrial Engineering, \\ IIT, Roorkee, Uttarakhand, India
}

\begin{abstract}
Bicycles are a global product and are present in every culture of the world. They are extensively used for travelling, they are pollution free and easy to manufacture. However, the stability is a great problem of concern while riding a bicycle. A two-wheeler is bound to fall after being tilted more than a certain angle of tilt. This paper aims to provide a literature review of the previously methods of achieving self-balancing condition in a bicycle. Further, up to $\mathbf{4}$ methods or de-signs have been broadly classified in this paper taking into account the previously published papers on self-balancing. Also, Gyroscopes are highly stabilizing de-vices which are used as stabilizing systems in ships and flight control of aircrafts. The goal of this paper is to finalize the best possible method for building a self-balancing twowheeler prototype capable of balancing itself using a gyroscope and PID (Proportional integral derivative) controller. The gyroscope will provide the necessary balance to the bicycle at the time of tilt. The idea of a selfbalancing bicycle can be further employed in the manufacture of driverless and driver operated automated two-wheelers respectively, with a high degree of road safety and road adhesion. Finally, this paper also covers the future scope and future techniques in the field of self-balancing
\end{abstract}

Keywords - Self Balancing, Bicycle, Gyroscope, Gyroscopic effect, Precession, PID controller, Balancing torque.

\section{INTRODUCTION}

Bi-cycles are a general routine form of transportation medium which can also be a medium of exercising. Over the years bicycles have served as an essential part of the human life as they have secondary functions as physical therapy for training of balance, power, endurance and synchronization. Hence, a bicycle is the very basic and simple example of the use of wheels and gear trains. Although riding a bicycle is meant to be a fairly simple task for the majority of people, this may not be the circumstance for some other people like for the young ones and for the adults who have failed to learn to ride a bicycle, injured people, or people having some form of cognitive disabilities. So here the necessity arises for a system that could act as a balancing aid to the bicycle rider.
Generally, a bi-cycle consists of the following: Frame, Drive train and gearing Handle bars, seating arrangements, Brakes, Suspension, Wheels and tyres and Other Accessories.

Now, From the mechanical point of view, about $99 \%$ of the power generated by the rider onto the pedals is transferred to the wheels for a non-gear cycle, and for a geared cycle this percentage is about 10 to $15 \%$ lessened or reduced.

\section{Problem Statement}

Nowadays, the general accident data for the world shows that the maximum road accidents occurring are of the two wheelers. And, Needless of the lot of investment is being done into the manufacturing and Research and development of high quality and technology driven motor bikes none have been seen to guarantee road safety to the motor rider and the Pillion rider. The safety here solely depends on the rider's skills and driving techniques. Also, road accidents are bound to occur as the person riding the bike loses control over it and the bike falls below its actual and theoretical point of fall. So here the necessity arises for a system that could act as a balancing aid to the bicycle rider.

\section{A. Problems in learning to balance}

- Pedalling causes the most complicated and worst imbalance situation because each push on a pedal is off centre in relation to the centre line of the bicycle. Hence this results in the generation of a maximum force which is to and fro in nature.

- Lateral instability is easier to learn and manage because of its fairly steady and unchanging nature as compared with the complicated and constantly changing forces due to pedalling. Also, the bicycle tyre widths are narrow and therefore they provide very less lateral stability.

- Centrifugal force, it occurs whenever the bicycle turns, i.e., ceases to go in a straight line.

- Forward/backward is then a factor arising as problem in learning bicycle though it is a very less significant factor as compared to the ones mentioned above. 


\section{International Journal of Engineering Applied Sciences and Technology, 2021 \\ Vol. 6, Issue 1, ISSN No. 2455-2143, Pages 228-234 \\ Published Online May 2021 in IJEAST (http://www.ijeast.com)}

\section{OBJECTIVE}

Although balancing of a two-wheeled vehicle is a challenging task. The objective of the paper is to review and develop a conceptual prototype that could provide balancing assistance to a bicycle rider without otherwise affecting the experience of riding a bicycle that could provide great benefit to the society. Such a system could be used both as a teaching tool, and as a physically therapeutic device. The project aims to design and build a bicycle prototype that is capable of balancing itself.

The paper focuses on the previously developed concepts of designing and developing a self-balancing two wheeler. And it is seen that the concept prototype deals with an experiment to produce gyroscopic effect on the prototype. The prototype will be a two wheel vehicle in which rotating discs mounted will act as a gyroscope to produce a counter balancing force, i.e., gyroscopic effect when the vehicle prototype will lose balance on either side. Thus, the vehicle will stabilize itself. Wherein even if an external force will be applied to the system the gyro sensors deployed in it will sense the force and will develop a force of similar magnitude but in opposite direction due to presence of gyroscopes used in the vehicle, thus the vehicle will not lose its balance even if the external force will be applied to it.

\section{B. Bi-Cycle Dynamics}

If the bicycle and rider are to be considered as a single system, then the forces that act on such a system and its components can be segmented into two major groups namely: internal and external. The external forces are due to gravity, inertia, contact with the ground, and contact with the atmosphere. The internal forces are caused by the rider and by interaction between components [14].

The Fig.1 Here, depicts the to be balanced forces (dynamic natured) in a bicycle system.

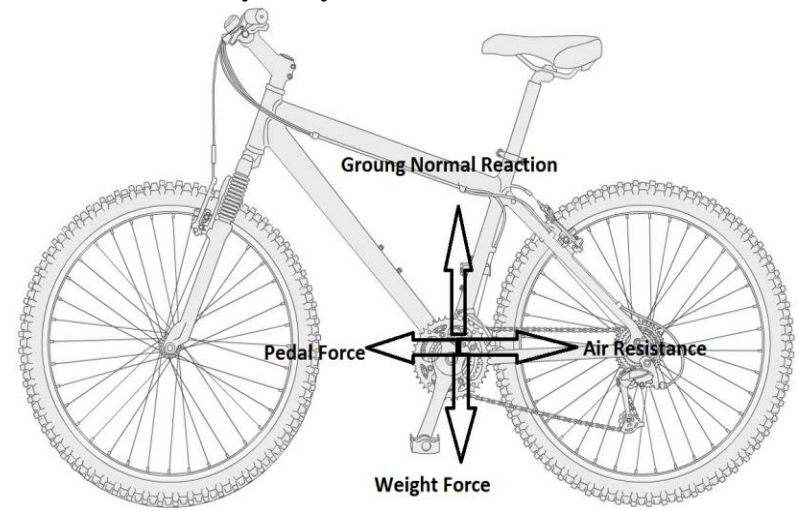

Fig. 1 Bicycle Dynamics (Force Considerations)

\section{Previous Research Reviews}

\section{Balancing Using Flywheel}

Such a prototype is divided into two parts. First is the balancing control and second is tracking control. In this approach a bicycle robot was developed whose balancing is achieved using flywheels. Here, the direction of the flywheel is guided and which in turn controls the bicycle position. Also, a navigation system is implemented onto this robot. Such a prototype also uses a compass and an encoder to check the required direction and the steering position respectively. Then an encoder sensor is also added to detect the flywheel position and to control the flywheel to be in horizontal position. Flywheel's angle and the bicycle's tilt angle is further controlled using PID control. During balancing situations, the flywheel is used as an actuator where encoder and Gyroscope are the sensors. Flywheel encoder is used to monitor the flywheel position whereas Gyroscope is used to sense the Bicycle leaning position. Hence the balancing of the bicycle takes place [12].

\section{Balancing Using Rotating Discs}

The aim of this prototype is to balance a two wheeler against any impact and in zero velocity as well i.e. under idling conditions when the engine or motor is running whereas the vehicle is stationary at a standstill. Here, two heavy rotating disks are employed around the frame in order to compensate the tilt of the vehicle and make it stable. The tilt angle of the chassis is measured by an android device using orientation sensor. The input is then sent to a Bluetooth receiver which is connected to an Arduino. The angle of tilt then acts as the input data that is taken by an android device. The device then further sends a control signal to the Arduino accordingly. Using the inputs, tilt direction of the rotating disk is balanced by controlling the motor from the Arduino. Hence, achieving the desired balancing of the bicycle [13].

\section{Balancing Using a Stabilizing Wheel (Gyroscope Approach)}

In this approach the bicycle is considered as an inverted pendulum. Now, to balance a rider-less bicycle, a stabilizing wheel is used and an IMU (Inertial Measurement Unit) to ensure that the bicycle is always in the upright position and balanced. The tilt and angular velocity are measured along with the speed of the stabilizing wheel. The direction of movement of the stabilizing wheel compensates the tilt in the either direction. If the bicycle tilts towards left, the stabilizing wheel rotates in the clockwise di-rection and if the bicycle tilts to the right, the stabilizing wheel rotates anticlockwise.

The bicycle wheel uses a gyroscope. It uses common bicycle wheel, which reduces cost with a great amount. After installing this wheel the ground clearance is also reduced. The arrangement of the stabilizing wheel makes it a very odd structure to ride. The structure very efficiently balances the bicycle but makes it very difficult to ride. The size of the stabilizing wheel is a major drawback to this structure but can be reduced by replacing the material of the wheel used. Instead of using heavy steel wheel, a Tungsten, Osmium, or Lead disk can be used which can fit inside the frame. But still the design looks very odd and makes it very difficult to ride. 


\section{International Journal of Engineering Applied Sciences and Technology, 2021 Vol. 6, Issue 1, ISSN No. 2455-2143, Pages 228-234 \\ Published Online May 2021 in IJEAST (http://www.ijeast.com)}

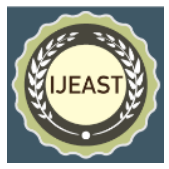

\section{Research Papers Reviewed}

The main goal of this literature review is to present an idea of getting familiar with the various methods and designs developed till date for achieving self balancing of a bicycle or a two-wheeler.

Pallav Gogoi et al. designed and fabricated a small model or prototype of a bicycle or a two wheeler by means of a gyroscope. Their designed prototype works on the principle of inverted pendulum (or gyroscopic principle) and utilizes some electromechanical components for the balancing and controlling purposes. In their model they used a DC motor as the power train (which powers the gyroscope not the twowheeler wheels) along with the following parts: Steel Frame, 12V DC Motor, Mild steel disc, Rubber Wheels, Flat head stud, Nuts and bolts of M3and M size, Hub, Ball Bearings and Circlips. Here, they used a metallic disc placed at the centre point of the model at place of the rider seat, which in turn acts a gyroscope and provides the necessary gyroscopic balancing effect to the two-wheeler. Also, the COG (Centre of gravity) of the model was kept as low as possible just below the gyroscope so as to get better and high amount of grounding force or reaction, which is further better towards generating a constant and balanced contact of wheels. Finally, once the motor is started, the metallic MS (mid steel) disc fitted on the motor shaft starts to spin or revolve on its axis and gradually increases its angular velocity. Which then leads to the inductance of the gyroscopic couple effect and, whenever the two-wheeler loses its balance due to the active gyroscopic couple, an opposite reactive gyroscopic couple is generated due to gyroscopic effect, hence stabilizing the body. And during the working of the model, they found that after few rotations of DC motor connected disc, the motor and frame were under a stationary position and the gyroscope is said to be in absolute rolling motion about the spin axis. Further, they also performed graphical RPM (Revolution per minute) analysis along with varied weights of the gyroscopic arrangement at different motor speeds of their prototype so as to get the verification for the workability of their proposed design [1].

Ajith Gopinath et al. have designed a small scaled (sized) selfbalancing two-wheeler using gyroscope in their manuscript without a steering mechanism. Their proposed model of the two-wheeler consists of the following members: a flywheel, a floor-board, 2 sets of bearings, battery for power, 2 DC Motors, wheels and a voltage manipulation unit. The flywheel designed by them was made of ABS plastic so as to reduce the overall weight of the model. The 3D model presented by the authors was prepared on sketch up and solid edge softwares whereas the analysis of the flywheel was performed on ABAQUS software. Here, they used a PID controller in order to measure the displacements in the vehicle linear and angular velocities, which are then sent to the control input. As far as the working of the model is concerned, a flywheel can be considered as a capacitor as it also stores and releases rotational energy as and when there is a deficient in a cyclic manner same as a capacitor stores and re-leases positive power or voltage. And, during high RPMs the flywheel can induce residual stresses and store mechanical energy while at low RPMs it will not give the twowheeler the required force to stabilize. They further observed that at low RPMs till about 1500 no stabilization was achieved whereas at 2000 some partial stabilization was seen and at RPM of about 2500 the prototype was under a stable linear motion. Also, as the prepared model is of a relatively small size when compared to an actual bicycle or two-wheeler at about 3500 RPM the assemblies tend to loosen their strength and at 4000 RPM and above the flywheel assembly were completely disassembled [2].

Rahul Kumar et al. in their paper titled "Models for SelfBalancing of Two Wheeled Vehicles: A Review", reviewed the various existing methods and research findings in the field of self balancing two-wheeler and concluded that while all the proposed designs said to have achieved the self balancing, mostly using a PID controller or a Bluetooth signal sending device with a gyroscope there was no evident finding in them as they all lacked either the maximum rider weight or the size of the proposed model was too small. And evidently the existing designs or models have a number of drawbacks which are currently in need of further improvement. Also, they proposed two of their own designs/models the first being the balancing by providing an opposite thrust of tilt to the vehicle (twowheeler). In their proposed model, the servo motors is attached to a chain mechanism from one end and the other end is attached to a sprocket which is in the same axis as of the axis of the handle. Here, the movement of the sprocket turns the handle in the desired direction. A PID controller is used for input data and output. As, the motors rotate in the any direction causing the movement of the sprocket which will then turn the handle of the bicycle in the opposite direction. In other words, if the bicycle is turning left, the motor will move in such a way that it will turn the steering handle towards right and vice-versa. Also, the COG of the model vertically acts above the axis of the wheels at all times. In this way the model will always be in upright orientation and will not topple or fell over. And, this model has a high efficiency, light weight and low fabrication cost when compared to the previous existing models. The second proposed model for achieving self balance is done by adjusting the COM (center of mass) with respect to the motional tilt. Here, a conveyor belt is sup-posed to be used to which external weights are to be attached. And this mechanism is mounted on a rod just below the bicycle seat so it will fix the position of COM. Now, the weights will assist in the shifting of the overall total weight on either side. As a result of such a system the COM will transfer to the right under conditions where the bicycle tilts towards right and vice-versa. The aim of this system is to transfer the loads on the conveyor belt in opposite to the incline in order to keep the COM fixed below the rider seat [3]. 


\section{International Journal of Engineering Applied Sciences and Technology, 2021 Vol. 6, Issue 1, ISSN No. 2455-2143, Pages 228-234 \\ Published Online May 2021 in IJEAST (http://www.ijeast.com)}

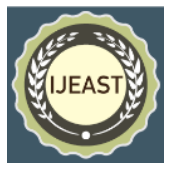

N. Tamaldin et al. reviewed 5 research papers on the self balancing of a two-wheeler. According to them there are two situations wherein a bicycle can tend to fall the first situation is when a bicycle tends to fall under relatively low transitional velocities. And the second situation is when there are steering situations i.e. the handle of the bicycle is rotated so as to make a right or a left turn. Also, in their paper they listed 4 so far possible methods of achieving the self balancing condition. These methods are self balancing using a control moment gyroscope (CMG) (high torque providing method), self balancing using mass balancing technique (low torque providing meth-od), self balancing by achieving steering or handle control (low energy needing and less weighted), self balancing using reaction wheel (high energy needing and low torque developing method). Further, they concluded that out of the above mentioned 4 methods the steering control fails under low transitional velocities and the others also have limitations when it comes to practical application and working except the CMG method. Finally, they found that the CMG method is the most apt one for achieving self balancing of a two-wheeler as its reaction period is relatively short, works perfectly at low transitional velocities and when the bicycle is at stationary position, has zero vertical ground reaction forces and on the top of it all can generate high magnitudes of stabilization torques. However, this method also lacks in terms of complex construction/assembly and fitment; and consumes a high amount of DC power or energy therefore it requires further development in its design [4].

Jiarui He et al. in their paper named "Control System Design of Self-balanced Bicycles by Control Moment Gyroscope" designed a self balancing two-wheeler prototype using a CMG system. And they obtained a possible feedback coefficient of the system by MATLAB simulations. They mainly classified the methods to achieve self-balance into 4 categories/types. Back in 1998 the very 1st method proposed was by using a simple gyroscope mechanism; in 2014 a sliding mode controller (SMC) was utilized to control a CMG system, and it is seen that this CMG provides a large torque generating but high energy consuming system. The 2 nd type is by using mass balancing by swinging a massive ball, while the mechanical construction of mass balancing is simple; the torque generated in this method is very small. The 3rd type is achieved by using steering control and as mentioned above this method however consuming less energy does not achieves balancing under low vehicle velocities. And finally, the 4th type uses a reaction wheel which has a short response time, but has a limited output torque, therefore it is only suitable for a small sized bicycle. They also fabricated a self-balancing prototype using a CMG system, wherein the CMG mechanism is set and assembles at the back seat of the bicycle. Now, for the working of the self balancing model they considered the relative motion of bicycle in three parts, here the body is considered to rotate in the $\mathrm{x}$-axis with a lean angle; the gyroscopic gimbals and flywheel are to rotate in $\mathrm{y}$-axis with a precession angle; the flywheel rotate in $\mathrm{z}$-axis.
They have also mentioned the MATLAB simulation matrix equations in their manuscript. And at the end they have concluded a combination system of steering control mechanism with the CMG system so as to reduce the energy consumption and utilize twin gyroscopes for eliminating the effect of the vertical unnecessary reaction torque [5].

Sergio Tamayo et al. utilized a single gimbal CMG system for the production of the balancing torque which reacts in opposite direction to the gravitational tilting moment. According to them this system has the following advantages: a) it generates substantial torques corresponding to small gimbal torque inputs, b) it is DC power operated, iii) it has zero reactional ground forces and iv) it can balance the bicycle at variable velocity conditions. Here, the balancing torque generated by the CMG system is said to act perpendicular to the flywheel torques. Further, they have reviewed the existing $\mathrm{CMG}$ balancing designs; and previously a State Feedback Control (SFC) was combined with a single gimbal CMG system which was placed just over the rear bicycle wheel. However, this design somewhat lacked stability which here is the most important factor of concern. Then self balancing models were developed using a Sliding Mode Control (SMC) system in combination with a single-gimbal CMG placed just beneath the bicycle chassis (frame). And it was evident that the SMC proved to have improved performance. Now, the proposed design by the authors of self balancing was to be made, so they used Lagrange's method for setting the coordinates and parameters for their system. Next, they performed many stabilization iterations for the optimization of the feedback control signalling system. And finally, they concluded by giving a cascade extended state feedback control scheme to solve the problem of stabilization and disturbance rejection of a bicycle with were faced in a CMG system stabilization unit or model. Also, their proposed scheme is said to be thoroughly validated by various simulations performed on model self balancing bi-cycle under pulsating lateral disturbance conditions on MATLAB and ADAMS soft-wares. And, the results achieved promised very high robustness, better disturbance rejection characteristics and a relatively faster and constrained stabilization response [6].

A. V. Beznos et al. explained that in a regular bicycle the front turning wheel also controls and affects the stability of the bicycle other than changing the direction. Also, they proposed three control loops for the control and stabilization of the motion of the gyroscopic bicycle during tilting situation. And they fabricated a kid sized Dc battery powered self balancing prototype. Here, each loop has its specific role. The 1st loop affects the steering angle i.e., the turning motion of the bicycle. As far as the working is concerned, an electronic encoder in combination with a rotary drive is installed on the steering handle to measure and alter the course. The 2nd servo loop controls the angular rotational velocity of the front wheel of the bicycle. Here, a separate encoder is fitted on the axis of the 


\section{International Journal of Engineering Applied Sciences and Technology, 2021 \\ Vol. 6, Issue 1, ISSN No. 2455-2143, Pages 228-234 \\ Published Online May 2021 in IJEAST (http://www.ijeast.com)}

wheel to determine the angular position and velocity. Also, the rear wheel here is left passive to move automatically due to the complete bicycle motion under friction. And finally, the 3rd loop controls the precession motion of the gyroscopes, hence resulting in curing the bicycle fall. In this, two additional encoders are inculpated: firstly, to quantify the orientation of gyroscopic system and secondly to determine the declination/tilt angle with respect to the general vertical.

Now, for the analysis purpose the gyroscope are free to move or rotate in 3 degrees of freedom (DOF). In terms of the constructional aspects the self balancing mechanism consists of 2 gyroscopes connected in a series to each other placed right between front and rear wheels of the bicycle. For the working a precession motion about the frame axes results in development of a gyroscopic torque which intern opposes the disbalancing/tilting torque under the gravity force. And finally, this stabilization control is achieved by use of 2 programmable controllers fabricated using the Intel 80C196KC chips having clock frequency of about $20 \mathrm{Mega} \mathrm{Hz}$ [7].

Sangduck Lee et al. designed an unmanned self balancing bicycle prototype by using a non-linear control system by virtue of the sliding patch and stuck phenomena (Mass balance). This method is the one that is previously discussed in the review section of the paper written by Jiarui He et al. in reference paper number 5. And, in their calculation and analysis for simplification they assumed that the load mass is located in middle of the mass balance mechanism. Further, they present a control strategy to change direction of the bicycle by moving the center of load mass respectively. In their paper, they derived a non-holonomic motion planning method with an easy kinematic and dynamic relation for their prototype. And this control strategy technique combines the managing of load mass of the bicycle with the organization of the steering angle and angular velocity of driving wheel for directional movement of bicycle. Also, one can find a couple of kinematic and dynamic modelling equations for the tilting conditions derived by considering gravitational and centrifugal effects in their paper including torques, radius of curvature for steering and tilt angles. Finally, the self balancing of the bicycle is achieved by the development of an opposing torque that is developed by the mass balance mechanism. However, for the practical working of the system they do not consider the backward driving situation i.e. only the forward driving situation is considered [8].

Mukesh Kumar Prasad et al. presented a research paper to design and put together a rider less bicycle prototype that is competent of self balancing itself. This prototype in turn is governed by an electric DC motor and a reaction wheel mechanism and this method of self balancing is already discussed by N. Tamaldin et al. and Jiarui He et al. in reference papers 4 and 5 respectively. Here, a sensor is installed in order to detect and store data about the angle of tilt of the vehicle and then send this sensor data to the control system which outputs a balancing torque to the motor powering the balancing reaction wheel. Also, in their design they have considered the condition of tilting of a bicycle with an inverted pendulum approach. And, an inverted pendulum is one in which its mass acts above its pivot point; an inverted pendulum is naturally unstable in its construction so it must be actively balanced in order to be in upright configuration. According to them this automatic balancing prototype is created by a combination of several geometry effects due to mass distribution, and forward motional speed. And the components which influence it are tyres, suspension system, steering handle and bearings, and frame (chassis). Further, as far as the construction and positioning of the balancing reaction wheel system is concerned it is exactly placed beneath the seat of the rider. However, the main limitation of this proposed design is that it does not permits easy steering, principally under high velocities. For the control mechanism an AT mega 16 microcontroller and for angle sensing a Triple Axis Accelerometer ADXL335 are employed respectively, here in this design. Then finally, same as the above reviewed designs here also an external balancing torque is responsible for the automatic balancing of the inline twowheeler during its motion and stationary situations [9].

\section{WORKING CYCLE FOR GYROSCOPIC SELF-BALANCING MODEL}

After going through the above-mentioned reviews, the final working cycle for the gyroscopic self balancing prototype is given below:

This working cycle consists of 5 sub processes or steps namely,

- Chassis tilt position or angle of tilt data

- Sensor data collection

- Arduino data reading

- Receiving of commands by motor driver circuit

- Motor unit torque generation

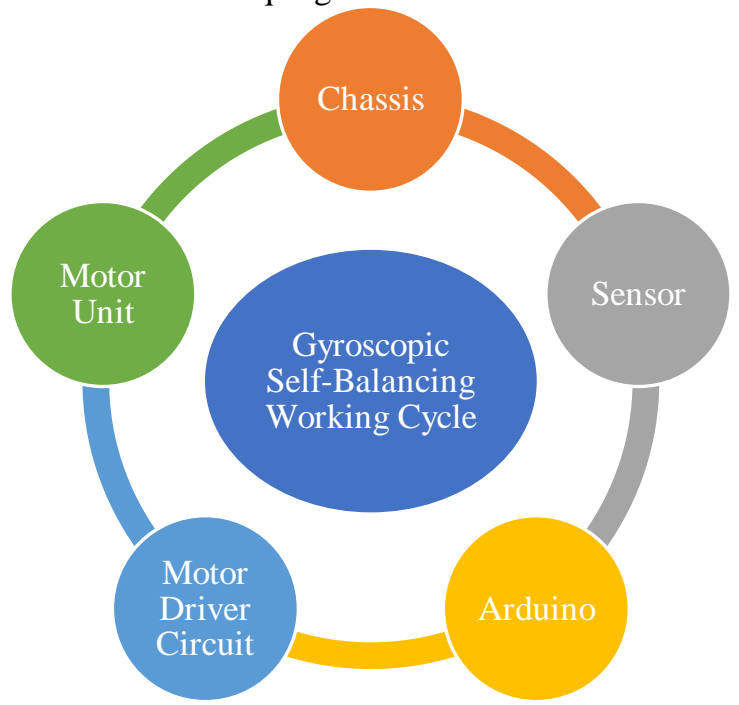

Fig. 2. Working Cycle 


\section{International Journal of Engineering Applied Sciences and Technology, 2021 \\ Vol. 6, Issue 1, ISSN No. 2455-2143, Pages 228-234 \\ Published Online May 2021 in IJEAST (http://www.ijeast.com)}

\section{Future SCOPE AND TECHNIQUES}

Gyrowheel/Gyrotyre

A Jyrobike is a bicycle manufactured by a London based company named Jyrobike limited, which designs and fabricates small kid sized self balancing bicycles and toy bikes; further these come with a special front wheel designed to demonstrate automatic balancing. Here, special front wheel contains a rotary flywheel motioned by a DC motor powered by a DC rechargeable battery, which spins like a gyroscope at high RPMs. And, the flywheel spins a lot faster than the front wheel rolling motion hence acting as a gyroscope, also under slow vehicle speeds. This type of a bike is said to use a gyro-wheel, which is nothing more than a simple precessing compact device which works just as a gyroscope and hence produces the required opposite balancing torques. In the constructional aspects a gyro-wheel is quite similar to a hub electric motor tyre which has internal copper winding [10].

Jyrobike technology is developed to help children and adults learn to ride a bicycle quickly and easily, this is basically for people lack confidence in their maintaining their balance during riding a bicycle.

However, like all the other methods and designs of self balancing bicycles this also has some disadvantages, first of them being that this can only be manufactured in small kid sizes only as large size would incur bulky, complex and less effective characteristics. Second disadvantage is that this jyrobike although achieving self balancing somewhere limits the ability of the rider to steer under tilting conditions. And finally, the third disadvantage of this type of a design is the limited speed balancing i.e. this design can work up to a certain speed only as it fails under higher denominations of transitional speeds say more than $40 \mathrm{~km} / \mathrm{hr}$ [11].

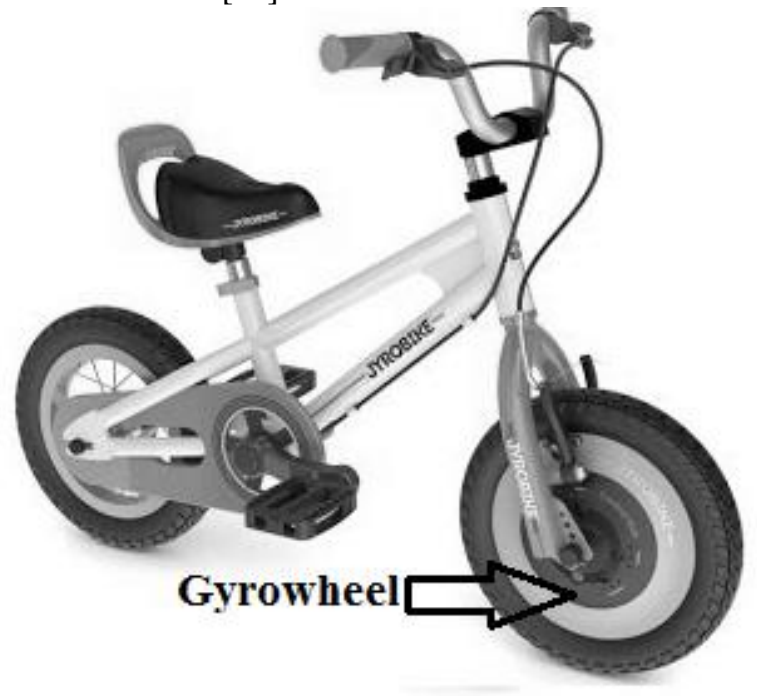

Fig. 3. Jyrobike

\section{CONCLUSION}

Now, after going through the above given knowledge and information about how to automatically balance a bicycle, we can finally conclude that the best and the most apt method for the achieving self-balance condition in a bicycle is by using the method suggested /proposed by Jiarui He et al. in their paper named "Control System Design of Self-balanced Bicycles by Control Moment Gyroscope", in the reference paper number 5. However, the CMG method has some limitations as well; the energy consumed by the system setup is very high. And also, as the CMG device consists of a vertical torque induction with respect to the bicycle, the front wheel of the bicycle might tend to leave the ground resulting in the bicycle falling in the backward direction.

\section{REFERENCE}

[1] Gogoi, P., Nath, M., Doley, B. T., Boruah, A., \& Barman, H. J. (2017) . Design and Fabrication of Self Balancing Two Wheeler Vehicle. In International Journal of Engineering and Technology (IJET). (pp.2051 - 2058).

[2] Gopinath, A., Rooby, A. T., \& Babu, S. (2017). Design and Fabrication of Self Balancing Two-Wheeler Model Using Gyroscope. In International Journal of Engineering, Technology, Science and Research, 9(3), 2051-2058.

[3] Kumar, R., Mathur, A., Rawat, T. S., \& Butola, R. Models for Self-Balancing of Two Wheeled Vehicles: A Review. In International Journal on Recent and Innovation Trends in Computing and Communication, 5(5), (pp.537-539).

[4] Tamaldin, N., Yusof, H. I. M., Abdollah, M. F. B., Omar, G., \& Rosley, M. I. F. (2017). Design self-balancing bicycle. In Proceedings of Mechanical Engi-neering Research Day, 2017, (pp. 160-161).

[5] He, J., \& Zhao, M. (2015). Control system design of selfbalanced bicycles by control moment gyroscope. In Proceedings of the 2015 Chinese Intelli-gent Automation Conference (pp. 205-214).

[6] Tamayo-León, S., Pulido-Guerrero, S., \& Coral-Enriquez, H. (2017, October). Self-stabilization of a riderless bicycle with a control moment gyroscope via model-based active disturbance rejection control. In 2017 IEEE 3rd Colombian Conference on Automatic Control (CCAC) (pp. 1-6).

[7] Beznos, A. V., Formal'Sky, A. M., Gurfinkel, E. V., Jicharev, D. N., Lensky, A. V., Savitsky, K. V., \& Tchesalin, L. S. (1998, May). Control of autono-mous motion of two-wheel bicycle with gyroscopic stabilisation. In Proceedings. 1998 IEEE International Conference on Robotics and Au-tomation (Cat. No. 98CH36146) (Vol. 3, pp. 2670-2675).

[8] Lee, S., \& Ham, W. (2002, October). Self stabilizing strategy in tracking con-trol of unmanned electric bicycle with mass balance. In IEEE/RSJ interna-tional conference on intelligent robots and systems (Vol. 3, pp. 2200-2205).

[9] Prasad, M., \& Nirwan, N. W. (2016). Design and fabrication of automatic balancing bicycle. In International 
Journal of Science, Engineering and Tech-nology Research, 5(2), (pp.532-536).

[10] Wikipedia contributors. (2020, May 14). Jyrobike. In Wikipedia, The Free Encyclopedia. Retrieved 10:53, September 13, 2020, from https://en.wikipedia.org/w/index.php?title=Jyrobike\&oldi $\mathrm{d}=956690959$

[11] BASSO, D. K. (2008). Worldwide applications Patent No. PCT/US20 13/028083.

[12] Admin. (2013, October 23). Flywheel Balancing MachineTeaches you how to do balance. Retrieved from JP Balancer: https://www.jp-balancer.com/news/companynews-p1/425.html

[13] Hirano, T. (2001). United States Patent Patent No. US 6,189,371 B1.

[14] Soden, P.D. \& Adeyefa, Bodunrin. (1979). Forces applied to a bicycle during normal cycling.In Journal of biomechanics. (pp. 527-41). 